\title{
Reducing Crosstalk of Silicon-based Optical Switch with All- optical Multi-wavelength Regenerator
}

\author{
Feng Wen ${ }^{1,2}$, Yong Geng ${ }^{1}$, Mingle Liao ${ }^{1}$, Baojian Wu${ }^{1}$, Liangjun Lu ${ }^{3}$, Linjie Zhou ${ }^{3}$, Xingyu Zhou ${ }^{1}$, \\ Kun Qiu ${ }^{1}$, Jianping Chen ${ }^{3}$ \\ ${ }^{1}$ Key Lab of Optical Fiber Sensing and Communications, Ministry of Education, University of Electronic Science and Technology of China, \\ 611731 Chengdu, China \\ ${ }^{2}$ Aston Institute of Photonic Technologies, Aston University, B4 7ET Birmingham, UK \\ ${ }^{3}$ State Key Laboratory of Advanced Optical Communication Systems and Networks, Department of Electronic Engineering, Shanghai Jiao Tong \\ University, Shanghai 200240, China \\ fengwen@uestc.edu.cn
}

\begin{abstract}
Improving crosstalk performance of Mach-Zehnder-interferometer-type optical switches is experimentally investigated by use of an all-optical multi-wavelength regenerator. Extinction ratio and bit error rate of WDM signals are simultaneously improved in proposed regenerative optical switching.

OCIS codes: (190.4380) Nonlinear optics, four-wave mixing; (130.4815) Optical switching devices
\end{abstract}

\section{Introduction}

All-optical switching and regeneration as the core functions in high-capacity optical switching nodes have been widely studied. In recent years, silicon-based optical switches (OSs) draw intensive attention because of their complementary metal-oxide semiconductor (CMOS) compatibility, compact footprint and low cost. Mach-Zehnder interferometer (MZI)-type optical switches can provide broad operation bandwidth and high switching speed with electro-optic (EO) electrodes. 16×16 EO MZI-switches [1,2] and 32×32 thermo-optic (TO) MZI-switches [3] have been reported. However, high crosstalk limits their performance, especially for large-scale optical switches. Some methods, such as dilated architectures [4], adding additional attenuators [5] or semiconductor optical amplifiers (SOAs) [6] were proposed to improve the crosstalk performance by modifying switch structure. In this paper, we use the all-optical regenerator to reduce the crosstalk noise, which can be adopted by optical switches with various controlling mechanisms. To meet the requirement of wavelength-division multiplexed (WDM) systems, multiwavelength regenerators are also necessary in this regenerative optical switching. By analyzing the signal degradation due to the low isolation in the silicon-based optical switch, we experimentally investigate the crosstalk suppression of WDM signals using a data-pump four-wave mixing (FWM)-based regenerator. Extinction ratio (ER) and bit error rate (BER) are simultaneously improved for the four-wavelength optical return-to-zero (RZ) signals.

\section{Signal degradation by the in-band crosstalk}

For the MZI-type optical switch, the crosstalk is caused by the imbalance power splitting and attenuation in two parallel arms. Moreover, the switching principle of introducing optical phase shift using EO effect in one arm will also induce extra free-carrier absorption (FCA), which results in the further imbalanced attenuation and higher crosstalk in the "Cross" or "Bar" state. To investigate the signal degradation due to crosstalk, an optical RZ signal and a continuous wave (CW) light as in-band noise were input into the adjacent ports of a $4 \times 4$ silicon-based MZI optical switch. This MZI-type optical switch has both EO and TO tuners, which provides fast switching and phase error compensation, respectively [7]. The frequency of in-band noise is $192.2 \mathrm{THz}$, deviating from the RZ frequency of $192.1 \mathrm{THz}$, but is close enough to influence the signal quality. With the increase of the number of p-i-n diodes (PIN) for the state switching, the RZ signal will suffer from the crosstalk due to the imbalance in the two parallel arms. Fig. 1 shows the ER as a function of the number of PIN and the signal spectrum with the in-band noise. The ER deterioration of $1.83 \mathrm{~dB}$ is obtained because of the influence from the adjacent ports. To enhance the switching performance, the all-optical regenerator with the advantage of ER improvement should be used.
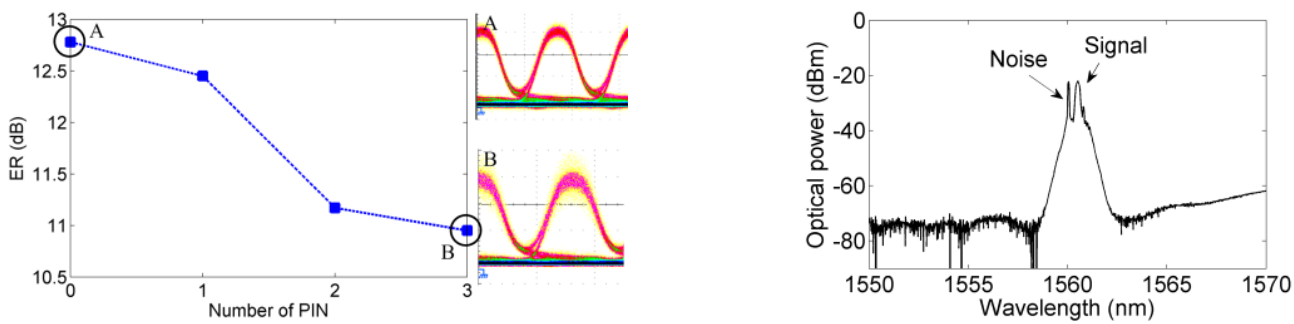

Fig. 1. The ER degradation with the number of PIN didoes and the optical spectrum of RZ signal with in-band noise. 


\section{Experiment and results}

An optical switching with all-optical regeneration is shown in Fig. 2. This experimental system includes four parts: optical signal generator, silicon-based optical switch, all-optical regenerator and optical receiver. The fourwavelength WDM-RZ signals with the frequencies of 192.1, 192.5, 192.9, 193.3THz were generated by cascaded modulation using $12.5 \mathrm{GHz}$ clock and $2^{31}-1$ pseudorandom-binary-sequence (PRBS) data. To achieve the proper pulse width for the multi-wavelength regeneration, the pulse compression was achieved by the phase modulator (PM) and the dispersion compensation fiber (DCF) in the multi-wavelength signal generator [8]. The in-band noise was generated by modulating a tunable laser with a nonreturn-to-zero (NRZ) signal. The frequency $\mathrm{f}_{\mathrm{N}}$ of in-band noise was fixed at $50 \mathrm{GHz}$ offset from the corresponding signal when it was tested for the switching and regeneration process.

The $4 \times 4$ Benes optical switch has three stages and six $2 \times 2$ MZI switch elements [7]. The initial "All-Bar" state was achieved by tuning the TO tuners to compensate the MZI arm imbalance. The WDM-RZ and in-band noise were respectively input into the $\mathrm{I}_{1}$ and $\mathrm{I}_{2}$ ports, and the output ports were $\mathrm{O}_{1}$ and $\mathrm{O}_{2}$. To investigate the worst case for the two-port input, three switching elements were changed from the "Bar" to "Cross" state and the output ports were $\mathrm{O}_{3}$ and $\mathrm{O}_{4}$. According to previous discussions, the WDM signals were influenced by the in-band noise.

The all-optical multi-wavelength regenerator handled the degraded WDM signals. Through the channel control unit including polarization controllers (PCs) and optical delay lines (ODLs), the required time interleaving and state of polarization (SOP) of the signals were obtained. The data pumps with the average power of about $27 \mathrm{dBm}$ were achieved by the high-power erbium-doped-fiber-amplifier (HP-EDFA) with the filter system. The four-wavelength all-optical regenerator is based on the data-pump FWM effects to improve the ER. The probe was a CW light at the frequency of $190.5 \mathrm{THz}$, and the probe power was fixed at $13 \mathrm{dBm}$. The nonlinear medium was a $1 \mathrm{~km}$-long highly nonlinear fiber (HNLF): the nonlinear coefficient $\gamma$ was $10 \mathrm{~W}^{-1} / \mathrm{km}$, the zero-dispersion wavelength $\lambda_{0}$ was $1556 \mathrm{~nm}$, and the dispersion slope at $\lambda_{0}$ was $0.0168 \mathrm{ps} / \mathrm{nm}^{2} / \mathrm{km}$. For the optical receiver, a $70 \mathrm{G} \mathrm{O/E}$ detector with an optical preamplifier was used to increase the sensitivity, and the signal quality was tested by the digital serial analyzer and error detector (ER).

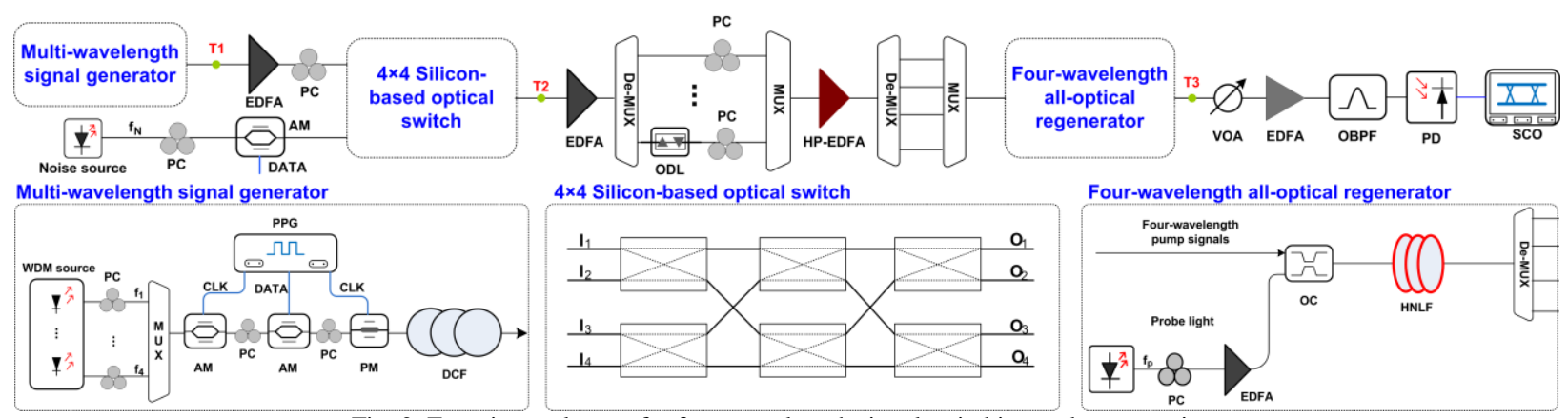

Fig. 2. Experimental setup for four-wavelength signal switching and regeneration.

Because the MZI has a broad operation bandwidth, the optical switching for the four-wavelength WDM signals covering $1.2 \mathrm{THz}$ frequency range can be achieved. However, the signal quality is degraded by the in-band noise, resulting in the low ER. The proposed data-pump FWM regenerator can improve the ER because of the slope of power transfer function (PTF) curve larger than one. Fig. 3 gives the Ch3 eye diagrams at input, switching and regeneration stages. It can be seen that the ER is deteriorated by $1 \mathrm{~dB}$ after the $4 \times 4$ optical switch, and improvement of $3.33 \mathrm{~dB}$ is achieved by the all-optical regenerator. ER improvements of other channels are 2.04, 1.57 and $1 \mathrm{~dB}$, respectively.

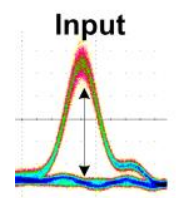

$E R=8.12 \mathrm{~dB}$
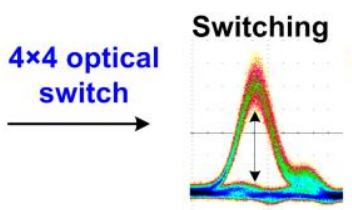

$E R=7.13 \mathrm{~dB}$

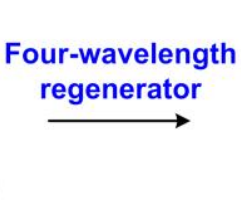

Regeneration

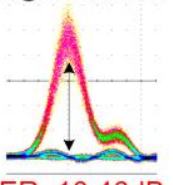

$E R=10.46 \mathrm{~dB}$

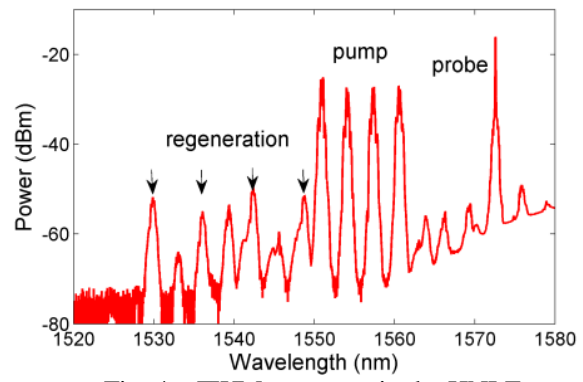

Fig. 4. FWM spectrum in the HNLF

Fig. 3. Eye diagrams of $\mathrm{Ch} 3$. 
By using the time-interleaving method, the nonlinear crosstalk in the multi-wavelength regenerator is reduced. The data-pump FWM spectrum is shown in Fig. 4. The high-order FWM products are sufficiently suppressed and the four-wavelength signals can be simultaneously regenerated. Fig. 5 gives the BER results of WDM signals and the sensitivity improvements are $2.65,1.07,3.07$ and $1.3 \mathrm{~dB}$, respectively. The different regeneration performances come from the different characteristics of input signals and PTF curves. The proposed regenerative optical switching scheme is also compatible to current NRZ-based communication systems through additional NRZ-to-RZ and RZ-toNRZ format conversion circuits.
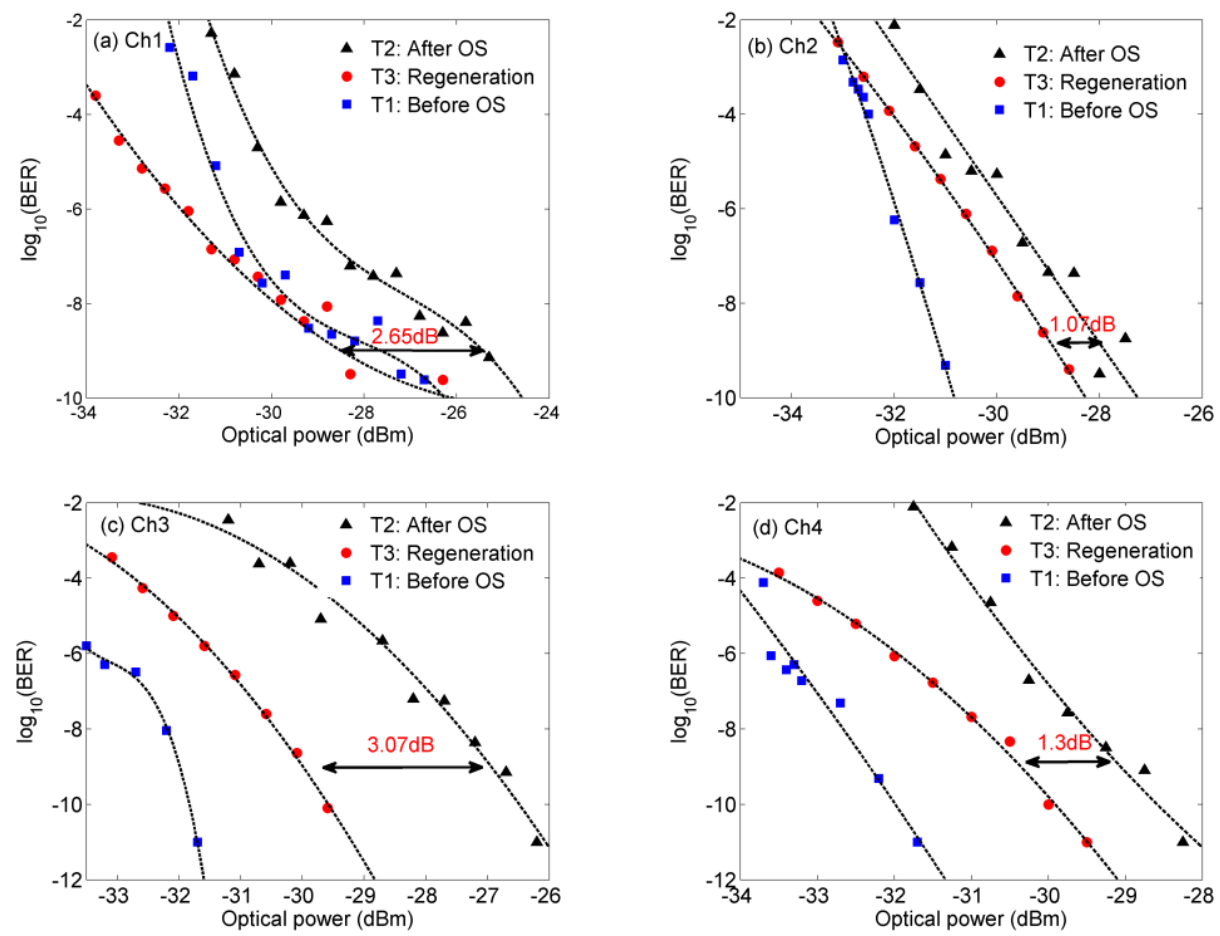

Fig. 4. BER results of four-wavelength signals.

\section{Conclusions}

We proposed a regenerative optical switching scheme to improve the crosstalk performance of silicon-based optical switches. We experimentally investigated the signal degradation due to the crosstalk of the MZI-type optical switch, and used the data-pump FWM-based multi-wavelength regenerator to improve the signal quality. ER and BER improvements were simultaneously achieved for the WDM signals.

\section{Acknowledgment}

This work has been supported by the National 863 Program of China (2013AA014402), the National Natural Science Foundation of China $(61505021,61671108)$, the Marie Sklodowska-Curie Action (701770-INNOVATION), the Fundamental Research Funds for the Central Universities (ZYGX2014J005), and the Program for Changjiang Scholars and Innovative Research Team in Universities of China (IRT1218), the 111 Project (B14039).

\section{References}

[1] L. Qiao, W. Tang, T. Chu, "16×16 non-blocking silicon electro-optic switch based on Mach-Zehnder interferometers," OFC, Th1C.2 (2016).

[2] L. Lu, S. Zhao, L. Zhou, et al., "16×16 non-blocking silicon optical switch based on electro-optic Mach-Zehnder interferometers," Opt Express 24, 9295-9307 (2016).

[3] K. Tanizawa, K. Suzuki, M. Toyama, et al., "Ultra-compact 32×32 strictly-non-blocking Siwire optical switch with fan-out LGA interposer," Opt Express 23, 17599-17606 (2015).

[4] K. Padmanabhan and A. Netravali, "Dilated networks for photonic switching," IEEE Trans Commun COM-35, 1357-1365 (1987).

[5] D. H. P. Maat, Y. C. Zhu, F. H. Groen, et al., "Polarization-independent dilated InP-based space switch with low crosstalk," IEEE Photon Technol Lett 12, 284-286 (2000).

[6] Q. Cheng, A. Wonfor, R. V. Penty, et al., "Scalable, low-energy hybrid photonic space switch,” J Lightw Technol 31, $3077-3084$ (2013).

[7] L. Lu, L. Zhou, Z. Li, et al., "Broadband $4 \times 4$ nonblocking silicon electrooptic switches based on Mach-Zehnder interferometers," IEEE Photon J 7, 7800108 (2015).

[8] X.-Y. Zhou, B.-J. Wu, F. Wen, et al., "Total date rate of multi-wavelength 2R regenerators for time-interleaved RZ-OOK signals," Opt

Express 22, 22937-22951 (2014). 